\title{
A Strong Law of Large Numbers for Weighted Sums of i.i.d. Random Variables under Capacities
}

\author{
Defei Zhang and Ping He \\ School of Mathematics, Honghe University, Mengzi 661199, China \\ Correspondence should be addressed to Ping He; hepingky@163.com \\ Received 16 February 2014; Accepted 3 June 2014; Published 16 June 2014 \\ Academic Editor: Abdel-Maksoud A. Soliman
}

Copyright (C) 2014 D. Zhang and P. He. This is an open access article distributed under the Creative Commons Attribution License, which permits unrestricted use, distribution, and reproduction in any medium, provided the original work is properly cited.

With the notion of independent identically distributed (i.i.d.) random variables under sublinear expectations initiated by Peng, a strong law of large numbers for weighted sums of i.i.d. random variables under capacities induced by sublinear expectations is obtained.

\section{Introduction}

The strong law of large numbers plays important role in the development of probability theory and mathematical statistics; many studies about the extension of it have been completed by many authors. For example, Chow and Lai [1], Stout [2], Choi and Sung [3], Cuzick [4], Rosalsky and Sreehari [5], Wu [6], Bai and Cheng [7], Bai et al. [8], and so forth investigated the almost sure limiting behavior of weighted sums of i.i.d. random variables. In fact, the additivity of probability and expectations is not reasonable in many areas of applications because many uncertain phenomena cannot be well modeled using additive probabilities or linear expectations (see, e.g., Chen and Epstein [9], Huber and Strassen [10], and Wakker [11]). In the case of nonadditive probabilities, Marinacci [12] proved several limit laws for nonadditive probabilities and Maccheroni and Marinacci [13] obtained a strong law of large numbers for totally monotone capacities.

Recently, motivated by the risk measures, superhedge pricing, and modelling uncertainty in finance, Peng [14] introduced the notion of sublinear expectation space, which is a generalization of probability space. Together with the notion of sublinear expectation, Peng also introduced the notions about i.i.d., $G$-normal distribution, and $G$-Brownian motion. Under this framework, the weak law of large numbers and the central limit theorems under sublinear expectations were obtained in the studies by Peng in $[15,16]$. Soon thereafter, Denis et al. [17] introduced the function spaces and capacity related to a sublinear expectation. Chen et al. [18] proved a strong law of large numbers for nonadditive probabilities.

A natural question is the following: can we investigate strong laws of large numbers for weighted sums of random variables under capacities? Indeed, the goal of this paper is to discuss the strong laws of large numbers for weighted sums of i.i.d. random variables under capacities. Under some assumptions, we obtain a strong law of large numbers for weighted sums of i.i.d. random variables under capacities.

The paper is organized as follows: in Section 2, we give some definitions and lemmas that are useful in this paper. In Section 3, we give our main results including the proofs.

\section{Preliminaries}

In this section, we present some preliminaries in the theory of sublinear expectations and capacities. More details of this section can be found in the studies by Chen et al. [18] and Peng [19].

Let $(\Omega, \mathscr{F})$ be a measurable space, and let $\mathscr{H}$ be the set of random variables on $(\Omega, \mathscr{F})$.

Definition 1. A sublinear expectation $\widehat{\mathbb{E}}$ is a functional $\widehat{\mathbb{E}}$ : $\mathscr{H} \rightarrow R$ satisfying the following:

(i) monotonicity: $\widehat{\mathbb{E}}[X] \geq \widehat{\mathbb{E}}[Y]$ if $X \geq Y$;

(ii) constant preserving: $\widehat{\mathbb{E}}[C]=C$ for $C \in R$; 
(iii) subadditivity: $\widehat{\mathbb{E}}[X+Y] \leq \widehat{\mathbb{E}}[X]+\widehat{\mathbb{E}}[Y]$;

(iv) positive homogeneity: $\widehat{\mathbb{E}}[\lambda X]=\lambda \widehat{\mathbb{E}}[X]$ for $\lambda \geq 0$.

Artzner et al. [20] showed that a sublinear expectation can be expressed as a supremum of linear expectations. That is, if $\widehat{\mathbb{E}}$ is a sublinear expectation on $\mathscr{H}$, then there exists a set (say $\mathscr{P})$ of probability measures such that

$$
\widehat{\mathbb{E}}[X]=\sup _{P \in \mathscr{P}} E_{P}[X], \quad-\widehat{\mathbb{E}}[-X]=\inf _{P \in \mathscr{P}} E_{P}[X], \quad \forall X \in \mathscr{H} .
$$

For this $\mathscr{P}$, following Huber and Strassen [10], we define a pair $(\bar{C}, \underline{C})$ of capacities denoted by

$$
\bar{C}(A):=\sup _{P \in \mathscr{P}} P(A), \quad \underline{C}(A):=\inf _{P \in \mathscr{P}} P(A), \quad \forall A \in \mathscr{F} .
$$

Obviously,

$$
\bar{C}(A)+\underline{C}\left(A^{c}\right)=1,
$$

where $A^{c}$ is the complement set of $A$.

It is easy to check that $\bar{C}$ and $\underline{C}$ are two continuous capacities in the sense of the following definition.

Definition 2. A set function $C: \mathscr{F} \rightarrow[0,1]$ is called a continuous capacity if it satisfies

(1) $C(\phi)=0, C(\Omega)=1$;

(2) $C(A) \leq C(B)$, whenever $A \subset B$ and $A, B \in \mathscr{F}$;

(3) $C\left(A_{n}\right) \uparrow C(A)$, if $A_{n} \uparrow A$;

(4) $C\left(A_{n}\right) \downarrow C(A)$, if $A_{n} \downarrow A$, where $A_{n}, A \in \mathscr{F}$.

Definition 3 (see Peng [19]).

Identical Distribution. Let $X_{1}$ and $X_{2}$ be two $n$-dimensional random vectors in $\mathscr{H}$. They are called identically distributed, denoted by $X_{1} \stackrel{d}{=} X_{2}$, if, for each measurable function $\varphi$ on $R^{n}$ such that $\varphi\left(X_{1}\right), \varphi\left(X_{2}\right) \in \mathscr{H}$, one has

$$
\widehat{\mathbb{E}}\left[\varphi\left(X_{1}\right)\right]=\widehat{\mathbb{E}}\left[\varphi\left(X_{2}\right)\right] .
$$

Independence. A random vector $Y:=\left(Y_{1}, \ldots, Y_{n}\right), Y_{i} \in \mathscr{H}$, is said to be independent of another random vector $X:=$ $\left(X_{1}, \ldots, X_{m}\right), X_{i} \in \mathscr{H}$, under $\widehat{\mathbb{E}}$ if, for each measurable function $\varphi$ on $R^{m} \times R^{n}$ with $\varphi(X, Y) \in \mathscr{H}$ and $\varphi(x, Y) \in \mathscr{H}$ for each $x \in R^{m}$, one has

$$
\widehat{\mathbb{E}}[\varphi(X, Y)]=\widehat{\mathbb{E}}\left[\widehat{\mathbb{E}}[\varphi(x, Y)]_{x=X}\right] .
$$

Remark 4. A sequence of random variables $\left\{X_{i}, i \geq 1\right\}$ is said to be i.i.d., if $X_{i} \stackrel{d}{=} X_{1}$ and $X_{i+1}$ is independent of $Y:=$ $\left(X_{1}, \ldots, X_{i}\right)$ for each $i \geq 1$.

The following lemma shows the relation between Peng's independence and pairwise independence in the study by Marinacci in [12].
Lemma 5 (see Chen et al. [18]). Suppose that $X, Y \in \mathscr{H}$ are two random variables. $\widehat{\mathbb{E}}$ is a sublinear expectation and $(\bar{C}, \underline{C})$ is the pair of capacities induced by $\widehat{\mathbb{E}}$. If random variable $X$ is independent of $Y$ under $\widehat{\mathbb{E}}$, then $X$ is also pairwise independent of $Y$ under capacities $\bar{C}$ and $\underline{C}$; that is, for all subsets $D$ and $G \subset R$,

$$
C(X \in D, Y \in G)=C(X \in D) C(Y \in G)
$$

holds for both capacities $\bar{C}$ and $\underline{C}$.

Borel-Cantelli lemma is still true for capacities $\bar{C}$ and $\underline{C}$ under some assumptions.

Lemma 6 (see Chen et al. [18]). Let $\left\{A_{n}, n \geq 1\right\}$ be a sequence of events in $\mathscr{F}$.

(1) If $\sum_{n=1}^{\infty} \bar{C}\left(A_{n}\right)<\infty$, then $\bar{C}\left(\bigcap_{n=1}^{\infty} \bigcup_{i=n}^{\infty} A_{i}\right)=0$.

(2) Suppose that $\left\{A_{n}, n \geq 1\right\}$ are pairwise independent with respect to $\bar{C}$; that is,

$$
\bar{C}\left(\bigcap_{i=1}^{\infty} A_{i}^{c}\right)=\prod_{i=1}^{\infty} \bar{C}\left(A_{i}^{c}\right) .
$$

If $\sum_{n=1}^{\infty} \underline{C}\left(A_{n}\right)=\infty$, then $\underline{C}\left(\bigcap_{n=1}^{\infty} \bigcup_{i=n}^{\infty} A_{i}\right)=1$.

\section{Main Results}

In this section, we give our main results including the proofs.

Theorem 7. Let $\left\{X_{i}, i \geq 1\right\}$ be a sequence of i.i.d. random variables in $\mathscr{H}$ satisfying $\bar{\mu}=\widehat{\mathbb{E}}\left[X_{1}\right], \underline{\mu}=-\widehat{\mathbb{E}}\left[-X_{1}\right]$ and for any $h, r>0$

$$
\widehat{\mathbb{E}}\left[e^{\left(h\left|X_{i}\right|^{r}\right)}\right]<\infty .
$$

Let $\left\{a_{n i}, 1 \leq i \leq n, n \geq 1\right\}$ be an array of constants satisfying

$$
\begin{array}{r}
A_{\alpha}=\limsup _{n \rightarrow \infty} A_{\alpha, n}<\infty, \quad A_{\alpha, n}^{\alpha}= \\
\quad \frac{\sum_{i=1}^{n}\left|a_{n i}\right|^{\alpha}}{n}, \\
(1<\alpha \leq 2) .
\end{array}
$$

Then, for $0<r \leq 1$, if

$$
\bar{\mu}\left(1-\limsup _{n \rightarrow \infty} \frac{\sum_{i=1}^{n} a_{n i}}{b_{n}}\right) \geq 0,
$$

we have

$$
\begin{aligned}
\underline{C}(\underline{\mu} & \leq \liminf _{n \rightarrow \infty} \frac{\sum_{i=1}^{n} a_{n i} X_{i}}{b_{n}} \\
& \left.\leq \limsup _{n \rightarrow \infty} \frac{\sum_{i=1}^{n} a_{n i} X_{i}}{b_{n}} \leq \bar{\mu}\right)=1,
\end{aligned}
$$

where $b_{n}=n^{1 / \alpha} \log ^{1 / r} n$. 
Moreover, for $r>1$, if $\bar{\mu}\left(1-\lim \sup _{n \rightarrow \infty}\left(\sum_{i=1}^{n} a_{n i} / b_{n}\right)\right) \geq 0$, we have

$$
\begin{aligned}
\underline{C}(\underline{\mu} & \leq \liminf _{n \rightarrow \infty} \frac{\sum_{i=1}^{n} a_{n i} X_{i}}{b_{n}} \\
& \left.\leq \limsup _{n \rightarrow \infty} \frac{\sum_{i=1}^{n} a_{n i} X_{i}}{b_{n}} \leq \bar{\mu}\right)=1,
\end{aligned}
$$

where $b_{n}=n^{1 / \alpha}(\log n)^{(1+(\alpha-1)(r-1)) /(1+\alpha(r-1))}$.

In order to prove Theorem 7 , we need the following lemma.

Lemma 8. Let $\left\{X_{i}, i \geq 1\right\}$ be a sequence of i.i.d. random variables satisfying (8), and let $\left\{a_{n i}, 1 \leq i \leq n, n \geq 1\right\}$ be an array of constants. Truncate $\left|X_{i}-\bar{\mu}\right|$ at $\Delta_{n}$ and denote $X_{n i}:=$ $\left(X_{i}-\bar{\mu}\right) I_{\left\{\left|X_{i}-\bar{\mu}\right| \leq \Delta_{n}\right\}}$. Suppose that the following conditions hold:

(1) $\left|a_{n i} X_{n i}\right| \leq C\left|X_{i}\right|^{\beta} / \log n$ a.s. $\bar{C}$, for some $0<\beta \leq r$ and some constant $C>0$;

(2) $X_{n i}^{2} \sum_{i=1}^{n} a_{n i}^{2} \leq u_{n}\left|X_{i}\right|^{\delta} / \log n$ a.s. $\bar{C}$, for some $\delta>0$ and some sequence $\left\{u_{n}\right\}$ of constants such that $u_{n} \rightarrow 0$.

Then,

$$
\bar{C}\left(\limsup _{n \rightarrow \infty} \sum_{i=1}^{n} a_{n i} X_{n i}>\varepsilon\right)=0 \quad \forall \varepsilon>0 .
$$

Proof. From the inequality $e^{x} \leq 1+x+(1 / 2) x^{2} e^{|x|}$ for all $x \in R$, we have

$$
\widehat{\mathbb{E}}\left[e^{t a_{n i} X_{n i}}\right] \leq 1+\frac{1}{2} t^{2} a_{n i}^{2} \widehat{\mathbb{E}}\left[X_{n i}^{2} e^{t\left|a_{n i} X_{n i}\right|}\right]
$$

for any $t>0$. Let $\varepsilon>0$ be given. We set $t=2 \log n / \varepsilon$ and obtain by (1) and (2) in Lemma 8 and (8) that

$$
\begin{aligned}
\widehat{\mathbb{E}}\left[e^{t a_{n i} X_{n i}}\right] & \leq 1+\frac{2 \log ^{2} n}{\varepsilon^{2}} a_{n i}^{2} \widehat{\mathbb{E}}\left[X_{n i}^{2} e^{t\left|a_{n i} X_{n i}\right|}\right] \\
& \leq 1+\frac{2 u_{n} \log n}{\varepsilon^{2}} \frac{a_{n i}^{2}}{\sum_{i=1}^{n} a_{n i}^{2}} \widehat{\mathbb{E}}\left[\left|X_{i}\right|^{\delta} e^{(2 / \varepsilon) C\left|X_{i}\right|^{\beta}}\right] \\
& \leq 1+\frac{2 C_{1} u_{n} \log n}{\varepsilon^{2}} \frac{a_{n i}^{2}}{\sum_{i=1}^{n} a_{n i}^{2}} \widehat{\mathbb{E}}\left[e^{C(\varepsilon)\left|X_{i}\right|^{\beta}}\right] \\
& \leq 1+\frac{\log n}{2} \frac{a_{n i}^{2}}{\sum_{i=1}^{n} a_{n i}^{2}} \\
& \leq \exp \left\{\frac{\log n}{2} \frac{a_{n i}^{2}}{\sum_{i=1}^{n} a_{n i}^{2}}\right\}
\end{aligned}
$$

for all large $n$. For the large $n$, it follows by the Markov inequality and (15) that

$$
\begin{aligned}
\bar{C}\left(\sum_{i=1}^{n} a_{n i} X_{n i}>\varepsilon\right) & \leq e^{-t \varepsilon \widehat{\mathbb{E}}}\left[e^{t \sum_{i=1}^{n} a_{n i} X_{n i}}\right] \\
& \leq \frac{1}{n^{2}} \prod_{i=1}^{n} \exp \left\{\frac{\log n}{2} \frac{a_{n i}^{2}}{\sum_{i=1}^{n} a_{n i}^{2}}\right\} \\
& =n^{-3 / 2} .
\end{aligned}
$$

Using Lemma 6, we have

$$
\bar{C}\left(\limsup _{n \rightarrow \infty} \sum_{i=1}^{n} a_{n i} X_{n i}>\varepsilon\right)=0 \quad \forall \varepsilon>0 .
$$

The proof is complete.

Proof of Theorem 7. The proof of (11) is similar to that of (12); we only prove (12). We denote $X_{n i}^{\prime}:=\left(X_{i}-\right.$ $\bar{\mu}) I_{\left\{\left|X_{i}-\bar{\mu}\right| \leq(\log n)^{\left.\delta_{1}\right\}}\right.}, X_{n i}^{\prime \prime}:=\left(X_{i}-\bar{\mu}\right) I_{\left\{\left|X_{i}-\bar{\mu}\right| \leq(\log n)^{1 / r}\right\}}$, and $X_{n i}^{\prime \prime \prime}:=$ $\left(X_{i}-\bar{\mu}\right) I_{\left\{(\log n)^{\delta_{1}} \leq\left|X_{i}-\bar{\mu}\right| \leq(\log n)^{1 / r}\right\}}$ for $1 \leq i \leq n$ and $n \geq 1$, where $\delta_{1}=1 /(1+\alpha(r-1))$. Denote $a_{n i}^{\prime}:=a_{n i} I_{\left\{\left|a_{n i}\right| \leq n^{1 / \alpha}(\log n)^{\delta_{2}}\right\}}$ and $a_{n i}^{\prime \prime}:=a_{n i}-a_{n i}^{\prime}$ for $1 \leq i \leq n$ and $n \geq 1$, where $\delta_{2}=(r-1) /(1+\alpha(r-1))$. Then,

$$
\begin{aligned}
\frac{\sum_{i=1}^{n} a_{n i}\left(X_{i}-\bar{\mu}\right)}{b_{n}}= & \frac{\sum_{i=1}^{n} a_{n i}^{\prime} X_{n i}^{\prime}}{b_{n}}+\frac{\sum_{i=1}^{n} a_{n i}^{\prime \prime} X_{n i}^{\prime}}{b_{n}} \\
& +\frac{\sum_{i=1}^{n} a_{n i} X_{n i}^{\prime \prime \prime}}{b_{n}}+\frac{\sum_{i=1}^{n} a_{n i} X_{n i}^{\prime \prime}}{b_{n}} \\
:= & A_{n}+B_{n}+C_{n}+D_{n} .
\end{aligned}
$$

For $A_{n}$, we will apply Lemma 8 to the random variable $X_{n i}^{\prime}$ and weight $b_{n}^{-1} a_{n i}^{\prime}$. Note that

$$
\begin{aligned}
\left|b_{n}^{-1} a_{n i}^{\prime} X_{n i}^{\prime}\right| & \leq \frac{n^{1 / \alpha}}{b_{n}(\log n)^{\delta_{2}}}\left|X_{i}\right|=\frac{\left|X_{i}\right|}{\log n} \quad \text { a.s. } \bar{C}, \\
X_{n i}^{\prime 2} \sum_{i=1}^{n} b_{n}^{-2} a_{n i}^{\prime 2} & \leq \frac{n^{(2-\alpha) / \alpha} \sum_{i=1}^{n}\left|a_{n i}\right|^{\alpha}}{b_{n}^{2}(\log n)^{(2-\alpha) \delta_{2}}} X_{n i}^{\prime 2} \\
& \leq \frac{A_{\alpha, n}^{\alpha}}{(\log n)^{(2+\alpha(r-1)) /(1+\alpha(r-1))}} X_{i}^{2} \quad \text { a.s. } \bar{C} .
\end{aligned}
$$

Hence, by Lemma 8, we have

$$
\bar{C}\left(\limsup _{n \rightarrow \infty} A_{n}>\frac{\varepsilon}{2}\right)=0 \quad \forall \varepsilon>0 .
$$

For $B_{n}$, we observe that

$$
\begin{aligned}
B_{n} \leq \frac{(\log )^{\delta_{1}}}{b_{n}} \sum_{i=1}^{n}\left|a_{n i}^{\prime \prime}\right| \leq & \frac{(\log n)^{(1+(\alpha-1)(r-1)) /(1+\alpha(r-1))}}{b_{n} n^{(\alpha-1) / \alpha}} \\
& \times \sum_{i=1}^{n}\left|a_{n i}\right|^{\alpha}=A_{\alpha, n}^{\alpha} \quad \text { a.s. } \bar{C} .
\end{aligned}
$$

Namely,

$$
\bar{C}\left(\limsup _{n \rightarrow \infty} B_{n} \leq A_{\alpha, n}^{\alpha}\right)=1 .
$$

By replacing $X_{i}$ by $\theta X_{i}(\theta>0)$, we have

$$
\limsup _{n \rightarrow \infty} B_{n} \leq \frac{A_{\alpha, n}^{\alpha}}{\theta} \quad \text { a.s. } \bar{C} \text {. }
$$


Letting $\theta \rightarrow \infty$, we have

$$
\bar{C}\left(\limsup _{n \rightarrow \infty} B_{n} \leq 0\right)=1
$$

For $C_{n}$, note that

$$
\begin{gathered}
\left|\frac{a_{n i} X_{n i}^{\prime \prime \prime}}{b_{n}}\right| \leq \frac{A_{\alpha, n}}{\log n}\left|X_{i}\right|^{r} \quad \text { a.s. } \bar{C}, \\
\frac{X_{n i}^{\prime \prime \prime 2} \sum_{i=1}^{n} a_{n i}^{2}}{b_{n}^{2}} \leq \frac{A_{\alpha, n}^{2}}{\log ^{2} n}\left|X_{i}\right|^{2 r} \quad \text { a.s. } \bar{C} .
\end{gathered}
$$

Then, by Lemma 8 , we have

$$
\bar{C}\left(\limsup _{n \rightarrow \infty} C_{n}>\frac{\varepsilon}{2}\right)=0 \quad \forall \varepsilon>0 .
$$

For $D_{n}$, assumption (8) implies $\sum_{i=1}^{n} \bar{C}\left(\left|X_{n}\right|>\log ^{1 / r}\right)<$ $\infty$. Hence, by Lemma $6, \sum_{i=1}^{n}\left|X_{n i}^{\prime \prime}\right|$ is bounded a.s. $\bar{C}$. It follows that

$$
\begin{aligned}
D_{n} \leq & \frac{\sum_{i=1}^{n}\left|a_{n i}\right| \sum_{i=1}^{n}\left|X_{n i}^{\prime \prime}\right|}{b_{n}} \\
\leq & \frac{A_{\alpha, n}}{(\log n)^{(1+(\alpha-1)(r-1)) /(1+\alpha(r-1))}} \\
& \times \sum_{i=1}^{n}\left|X_{n i}^{\prime \prime}\right| \longrightarrow 0 \quad \text { a.s. } \bar{C}
\end{aligned}
$$

as $n \rightarrow \infty$.

Thus,

$$
\bar{C}\left(\limsup _{n \rightarrow \infty} D_{n} \leq 0\right)=1
$$

On the other hand, $\forall \varepsilon>0$; and we note that

$$
\begin{gathered}
\bar{C}\left(\limsup _{n \rightarrow \infty} \frac{\sum_{i=1}^{n} a_{n i} X_{i}}{b_{n}} \geq \bar{\mu}+\varepsilon\right) \\
\leq \bar{C}\left(\limsup _{n \rightarrow \infty} \frac{\sum_{i=1}^{n} a_{n i}\left(X_{i}-\bar{\mu}\right)}{b_{n}}\right. \\
\left.\geq \bar{\mu}\left(1-\limsup _{n \rightarrow \infty} \frac{\sum_{i=1}^{n} a_{n i}}{b_{n}}\right)+\varepsilon\right) \\
=\bar{C}\left(\limsup _{n \rightarrow \infty}\left(A_{n}+B_{n}+C_{n}+D_{n}\right)\right. \\
\left.\geq \bar{\mu}\left(1-\limsup _{n \rightarrow \infty} \frac{\sum_{i=1}^{n} a_{n i}}{b_{n}}\right)+\varepsilon\right),
\end{gathered}
$$

and the condition $\bar{\mu}\left(1-\limsup _{n \rightarrow \infty} \sum_{i=1}^{n} a_{n i} / b_{n}\right) \geq 0$ : from (20), (24), (26), and (28), we conclude that

$$
\bar{C}\left(\limsup _{n \rightarrow \infty} \frac{\sum_{i=1}^{n} a_{n i} X_{i}}{b_{n}} \geq \bar{\mu}+\varepsilon\right)=0 \quad \forall \varepsilon>0,
$$

which implies

$$
\bar{C}\left(\limsup _{n \rightarrow \infty} \frac{\sum_{i=1}^{n} a_{n i} X_{i}}{b_{n}}>\bar{\mu}\right)=0 .
$$

Also,

$$
\underline{C}\left(\limsup _{n \rightarrow \infty} \frac{\sum_{i=1}^{n} a_{n i} X_{i}}{b_{n}} \leq \bar{\mu}\right)=1 .
$$

Similarly, considering the sequence $\left\{-X_{i}, i \geq 1\right\}$, from (29), we have

$$
\underline{C}\left(\limsup _{n \rightarrow \infty} \frac{\sum_{i=1}^{n} a_{n i}\left(-X_{i}\right)}{b_{n}} \leq \widehat{\mathbb{E}}\left[-X_{1}\right]\right)=1 .
$$

Note that $\underline{\mu}=-\widehat{\mathbb{E}}\left[-X_{1}\right]$. So

$$
\underline{C}\left(\limsup _{n \rightarrow \infty} \frac{\sum_{i=1}^{n} a_{n i} X_{i}}{b_{n}} \geq \underline{\mu}\right)=1 .
$$

Therefore, the proof of Theorem 7 is complete.

The following theorem shows that if the norming constant $b_{n}$ is stronger than that of Theorem 7 , then condition (8) in Theorem 7 can be replaced by a weaker condition.

Theorem 9. Let $\left\{X_{i}, i \geq 1\right\}$ be a sequence of i.i.d. random variables in $\mathscr{H}$ satisfying $\bar{\mu}=\widehat{\mathbb{E}}\left[X_{1}\right], \mu=-\widehat{\mathbb{E}}\left[-X_{1}\right]$ and for some $h, r>0$

$$
\widehat{\mathbb{E}}\left[e^{\left(h\left|X_{i}\right|^{r}\right)}\right]<\infty \text {. }
$$

Let $\left\{a_{n i}, 1 \leq i \leq n, n \geq 1\right\}$ be an array of constants satisfying (9) in Theorem 7. Then, for $0<r \leq 1$ and $\beta>0$, if $\bar{\mu}(1-$ $\left.\lim \sup _{n \rightarrow \infty}\left(\sum_{i=1}^{n} a_{n i} / b_{n}\right)\right) \geq 0$, we have

$$
\begin{aligned}
\underline{C}(\underline{\mu} & \leq \limsup _{n \rightarrow \infty} \frac{\sum_{i=1}^{n} a_{n i} X_{i}}{b_{n}} \\
& \left.\leq \limsup _{n \rightarrow \infty} \frac{\sum_{i=1}^{n} a_{n i} X_{i}}{b_{n}} \leq \bar{\mu}\right)=1,
\end{aligned}
$$

where $b_{n}=n^{1 / \alpha}(\log n)^{1 / r+\beta}$.

Moreover, for $r>1$ and $\beta>0$, if $\bar{\mu}(1-$ $\left.\limsup _{n \rightarrow \infty}\left(\sum_{i=1}^{n} a_{n i} / b_{n}\right)\right) \geq 0$, we have

$$
\begin{aligned}
\underline{C}(\underline{\mu} & \leq \limsup _{n \rightarrow \infty} \frac{\sum_{i=1}^{n} a_{n i} X_{i}}{b_{n}} \\
& \left.\leq \limsup _{n \rightarrow \infty} \frac{\sum_{i=1}^{n} a_{n i} X_{i}}{b_{n}} \leq \bar{\mu}\right)=1,
\end{aligned}
$$

where $b_{n}=n^{1 / \alpha}(\log n)^{(1+(\alpha-1)(r-1)) /(1+\alpha(r-1))+\beta}$. 
Proof. We can prove that Lemma 8 is also true except that (8) and (1) of Lemma 8 are replaced by (35) and the following condition.

$$
\left|a_{n i} X_{n i}\right| \leq v_{n}\left|X_{i}\right|^{\beta} / \log n \text { a.s. } \bar{C} \text {, for some } 0<\beta \leq r \text { and }
$$
some sequence $\left\{v_{n}\right\}$ of constants such that $v_{n} \rightarrow 0$.

For the case $0<r \leq 1$, we let $X_{n i}^{\prime}=X_{i} I_{\left\{\left|X_{i}\right| \leq\left(h^{-1} \log n\right)^{1 / r}\right\}}$ and $X_{n i}^{\prime \prime}=X_{i}-X_{n i}^{\prime}$ for $1 \leq i \leq n$ and $n \geq 1$. For the case $r>1$, we let $X_{n i}^{\prime}=X_{i} I_{\left\{\left|X_{i}\right| \leq(\log n)^{1 /(1+\alpha(r-1))}\right\}}, X_{n i}^{\prime \prime}=X_{i} I_{\left\{\left|X_{i}\right|>h^{-1} \log ^{1 / r} n\right\}}$, and $X_{n i}^{\prime \prime \prime}=X_{i} I_{\left\{(\log n)^{1 /(1+\alpha(r-1))}<\left|X_{i}\right| \leq\left(h^{-1} \log n\right)^{1 / r}\right\}}$. The rest of the proof is similar to that of Theorem 7 and is omitted.

Remark 10. If $\bar{\mu}=\mu=0$ in Theorem 9, then, for $0<r \leq 1$ and $\beta>0$, we have

$$
\frac{\sum_{i=1}^{n} a_{n i} X_{i}}{b_{n}} \longrightarrow 0 \quad \text { a.s. }
$$

where $b_{n}=n^{1 / \alpha}(\log n)^{1 / r+\beta}$. Moreover, if $r>1$, we have

$$
\frac{\sum_{i=1}^{n} a_{n i} X_{i}}{b_{n}} \longrightarrow 0 \text { a.s., }
$$

where $b_{n}=n^{1 / \alpha}(\log n)^{(1+(\alpha-1)(r-1)) /(1+\alpha(r-1))+\beta}$. Since if $\alpha>1$ and $r \geq 1$, then $r(\alpha-1) / \alpha(1+r)>0$ and $(1+(\alpha-1)(r-$ $1)) /(1+\alpha(r-1))<1 / r+r(\alpha-1) / \alpha(1+r)$, we also have

$$
\frac{\sum_{i=1}^{n} a_{n i} X_{i}}{b_{n}} \longrightarrow 0 \text { a.s., }
$$

where $b_{n}=n^{1 / \alpha}(\log n)^{1 / r+r(\alpha-1) / \alpha(1+r)}$. The result is similar to Theorem 2.2 of Bai and Cheng [7].

Remark 11. If $\bar{\mu}=\mu=0$ in Theorem 7, we can get the classical strong law of large numbers for weighted sums of i.i.d. random variables as follows:

$$
P\left(\lim _{n \rightarrow \infty} \frac{\sum_{i=1}^{n} a_{n i} X_{i}}{b_{n}}=0\right)=1 .
$$

\section{Conflict of Interests}

The authors declare that there is no conflict of interests regarding the publication of this paper.

\section{Acknowledgments}

The authors thank Professor Zengjing Chen for his helpful discussion and suggestions and the reviewers for their valuable comments and suggestions to improve the presentation of this paper. This work is partially supported by the National Science Foundation of China (11301160), the Scientific Research Foundation of Yunnan Province (2013FZ116), the Scientific Research Foundation of Yunnan Province Education Committee (2011C120), the Reserve Talents Foundation of Honghe University (2014HB0204), and the Curricula Construction Foundations of Honghe University (ZYDT1308, ZDKC1111).

\section{References}

[1] Y. S. Chow and T. L. Lai, "Limiting behavior of weighted sums of independent random variables," Annals of Probability, vol. 1, no. 5, pp. 810-824, 1973.

[2] W. F. Stout, Almost Sure Convergence, Academic Press, New York, NY, USA, 2nd edition, 1974.

[3] B. D. Choi and S. H. Sung, "Almost sure convergence theorems of weighted sums of random variables," Stochastic Analysis and Applications, vol. 5, no. 4, pp. 365-377, 1987.

[4] J. Cuzick, "A strong law for weighted sums of i.i.d. random variables," Journal of Theoretical Probability, vol. 8, no. 3, pp. 625-641, 1995.

[5] A. Rosalsky and M. Sreehari, "On the limiting behavior of randomly weighted partial sums," Statistics \& Probability Letters, vol. 40, no. 4, pp. 403-410, 1998.

[6] W. B. Wu, "On the strong convergence of a weighted sum," Statistics \& Probability Letters, vol. 44, no. 1, pp. 19-22, 1999.

[7] Z. D. Bai and P. E. Cheng, "Marcinkiewicz strong laws for linear statistics," Statistics \& Probability Letters, vol. 46, no. 2, pp. 105112,2000

[8] Z. D. Bai, P. E. Cheng, and C. H. Zhang, "An extension of the Hardy-Littlewood strong law," Statistica Sinica, vol. 7, no. 4, pp. 923-928, 1997.

[9] Z. Chen and L. Epstein, "Ambiguity, risk, and asset returns in continuous time," Econometrica, vol. 70, no. 4, pp. 1403-1443, 2002.

[10] P. Huber and V. Strassen, "Minimax tests and the NeymanPearson Lemma for capacities," The Annals of Statistics, vol. 1, no. 2, pp. 251-263, 1973.

[11] P. Wakker, "Testing and characterizing properties of nonadditive measures through violations of the sure-thing principle," Econometrica, vol. 69, no. 4, pp. 1039-1059, 2001.

[12] M. Marinacci, "Limit laws for non-additive probabilities and their frequentist interpretation," Journal of Economic Theory, vol. 84, no. 2, pp. 145-195, 1999.

[13] F. Maccheroni and M. Marinacci, "A strong law of large numbers for capacities," The Annals of Probability, vol. 33, no. 3, pp. 11711178, 2005.

[14] S. Peng, "G-Expectation, G-Brownian motion and related stochastic calculus of Itô type," in Stochastic Analysis and Applications: The Abel Symposium 2005, vol. 2 of Abel Symposia, pp. 541-567, Springer, Berlin, Germany, 2007.

[15] S. Peng, "Law of large numbers and central limit theorem under nonlinear expectations," In press, http://arxiv.org/ abs/math/0702358.

[16] S. Peng, "A new central limit theorem under sublinear expectations," In press, http://arxiv.org/abs/0803.2656.

[17] L. Denis, M. Hu, and S. Peng, "Function spaces and capacity related to a sublinear expectation: application to G-Brownian motion paths," Potential Analysis, vol. 34, no. 2, pp. 139-161, 2011.

[18] Z. Chen, P. Wu, and B. Li, "A strong law of large numbers for non-additive probabilities," International Journal of Approximate Reasoning, vol. 54, no. 3, pp. 365-377, 2013.

[19] S. Peng, "Nonlinear expectationsand stochastic calculus under uncertainty-with robust central limit theorem and G-Brownian motion," In press, http://arxiv.org/abs/1002.4546.

[20] P. Artzner, F. Delbaen, J. Eber, and D. Heath, "Thinking coherently," Risk, vol. 10, no. 1, pp. 68-71, 1997. 


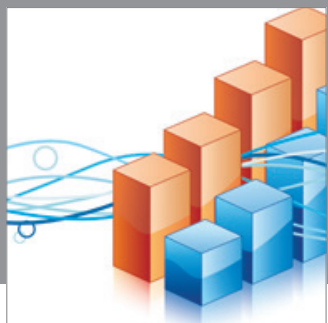

Advances in

Operations Research

mansans

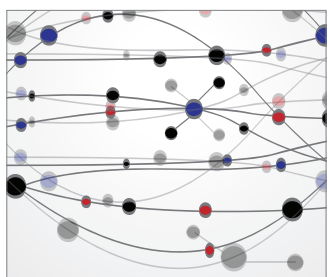

The Scientific World Journal
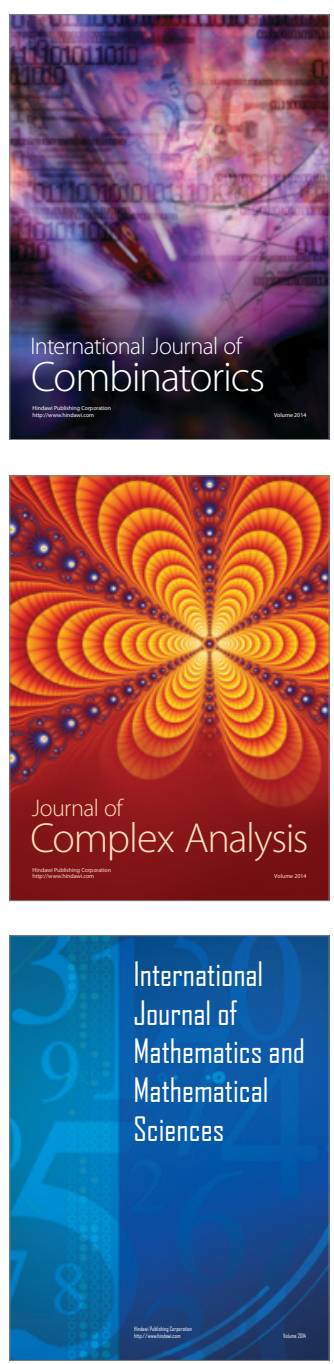
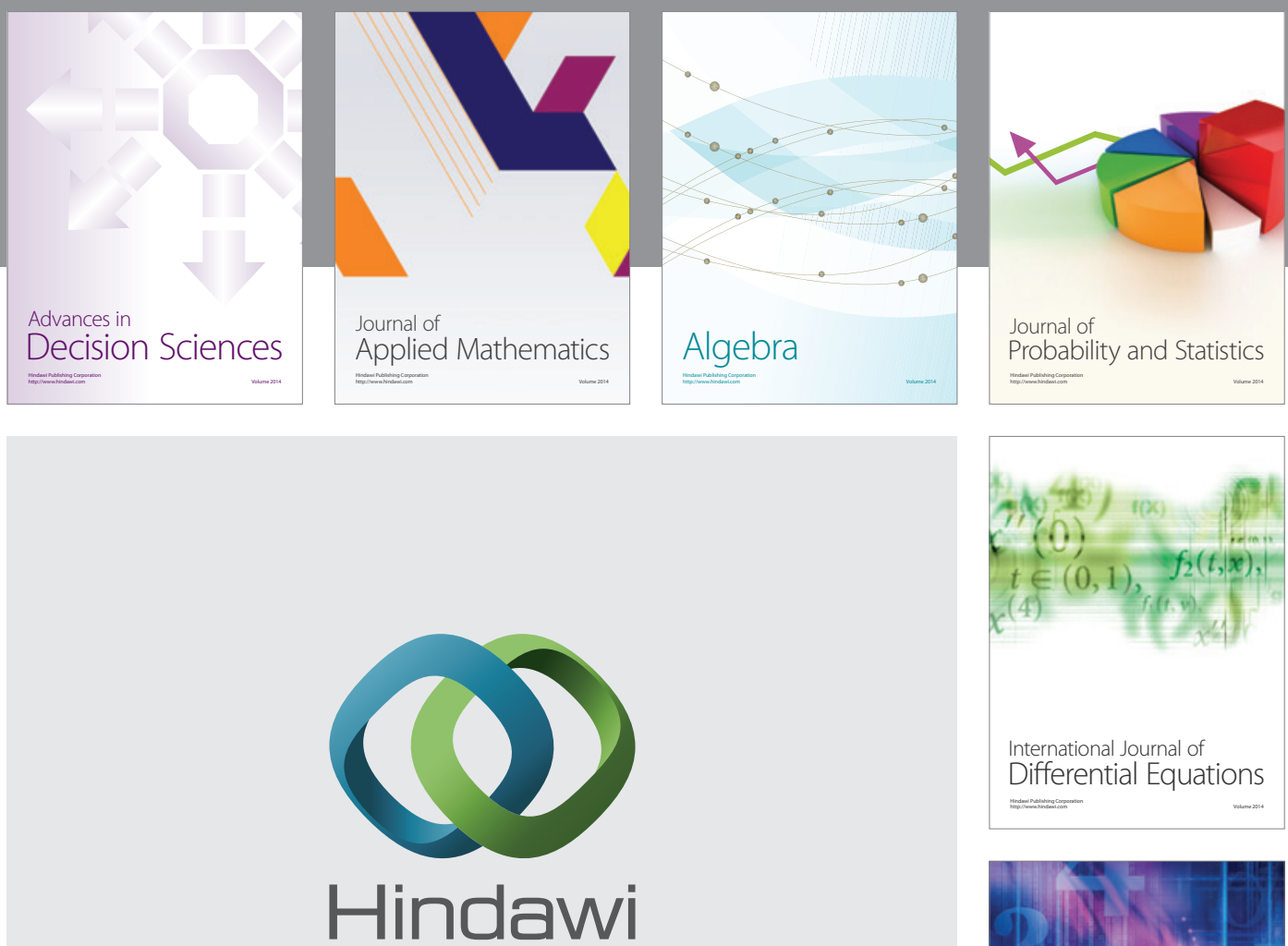

Submit your manuscripts at http://www.hindawi.com
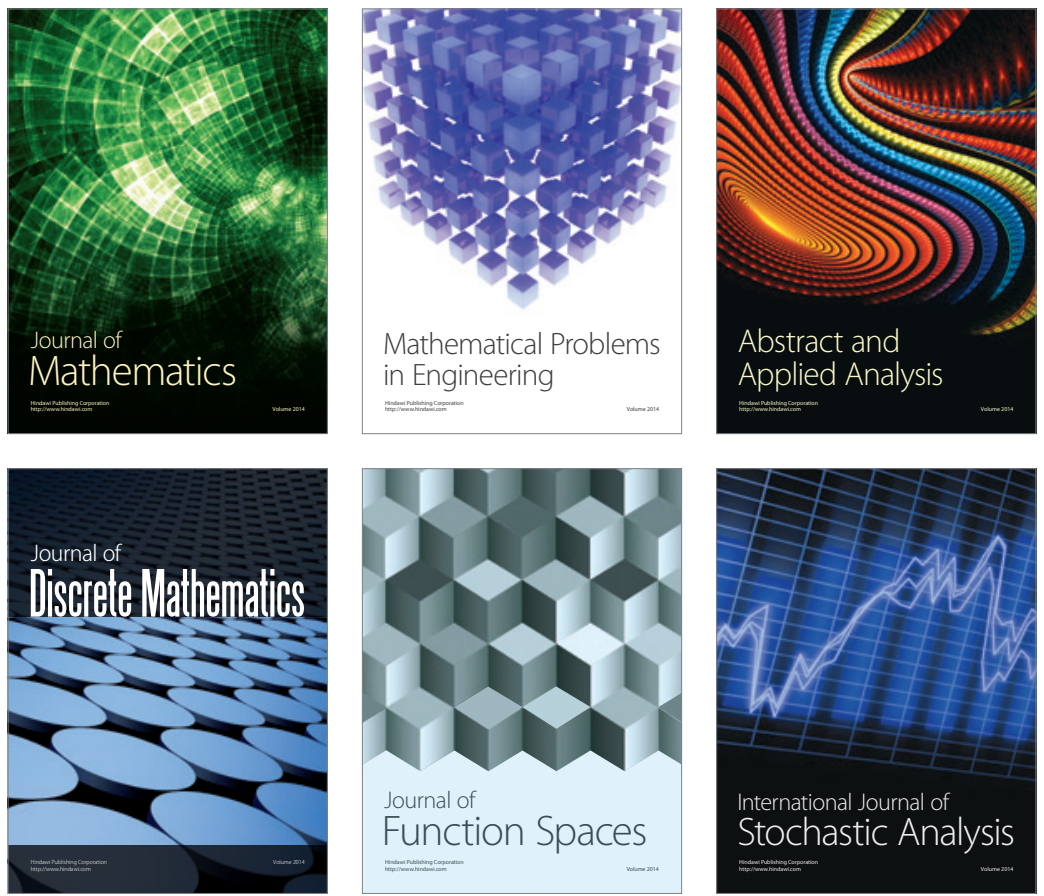

Journal of

Function Spaces

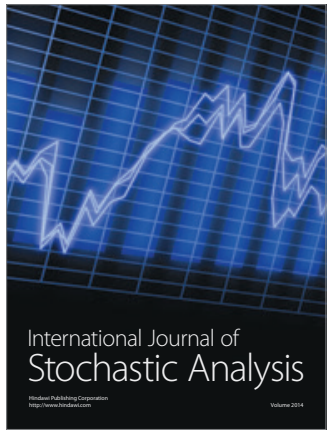

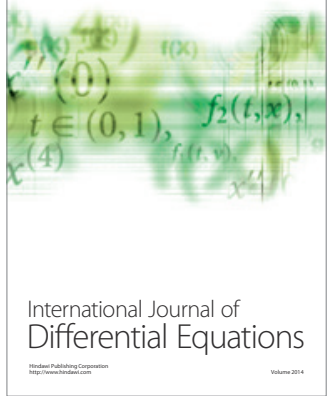
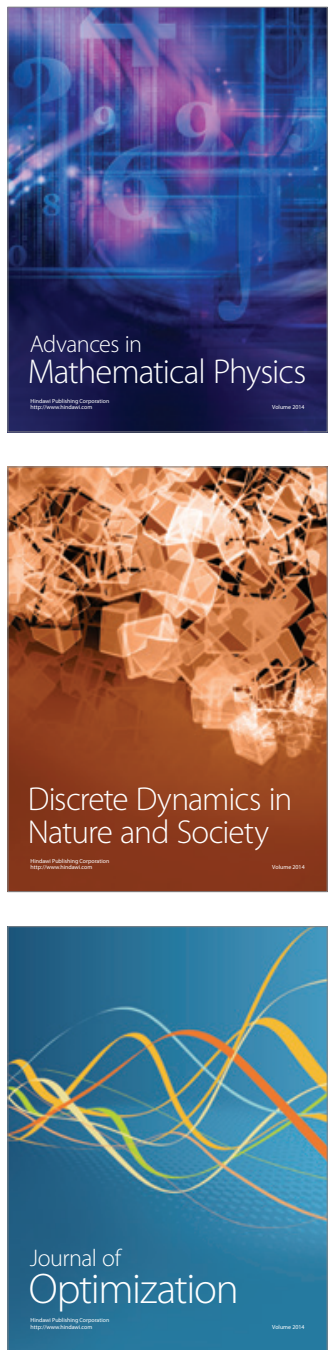